\title{
Arthroscopic Primary Repair of the Posterior Cruciate Ligament
}

\author{
Harmen D. Vermeijden, $\mathrm{MD}^{1} \quad$ Jelle P. van der List, $\mathrm{MD}^{1,2,3}$ Gregory S. DiFelice, MD ${ }^{1}$ \\ ${ }^{1}$ Department of Orthopaedic Surgery, Hospital for Special Surgery, \\ New York, United States \\ 2 Department of Orthopaedic Surgery, Spaarne Gasthuis Hospital, \\ Hoofddorp, The Netherlands \\ ${ }^{3}$ Department of Orthopaedic Surgery, Amsterdam UMC, University of \\ Amsterdam, Amsterdam Movement Science, Amsterdam, The \\ Netherlands \\ Address for correspondence Gregory S. DiFelice, MD, Hospital for \\ Special Surgery, 535 E. 70th Street, New York City, NY 10021 \\ (e-mail: difeliceg@hss.edu).
}

J Knee Surg 2021;34:478-485.

\author{
Abstract \\ Keywords \\ - posterior cruciate \\ ligament \\ - preservation \\ - primary repair \\ - arthroscopy
}

The posterior cruciate ligament $(\mathrm{PCL})$ is one of the four major stabilizers of the knee joint and functions as the primary restraint to posterior tibial translation. PCL tears rarely occur in isolation and most commonly presents in the setting of multiligamentous knee injuries. Several treatment strategies for these injuries have been proposed over the last decades, including ligament reconstruction and primary repair. Arthroscopic primary $\mathrm{PCL}$ repair has the potential to preserve native tissue using a more minimally invasive approach, thereby avoiding donor-site morbidity and allowing early mobilization. While arthroscopic PCL repair is certainly not an effective surgical approach for all patients, this procedure may be a reasonable and less morbid alternative to $\mathrm{PCL}$ reconstruction in selected patients treated for proximal or distal avulsion tears, with low failure rates, good knee stability, and good to excellent subjective outcomes. The surgical indications, surgical techniques, postoperative management, and outcomes for arthroscopic primary repair of proximal and distal $\mathrm{PCL}$ tears will be discussed in this review.
The posterior cruciate ligament (PCL) is one of the four major stabilizers of the knee joint and functions as the primary restraint to posterior tibial translation. ${ }^{1}$ Injury to the PCL rarely occurs in isolation and is most commonly present in the setting of multiligamentous knee injuries (MLIK) ${ }^{2-4}$ In patients with a complete PCL tear and symptomatic instability, surgery is typically preferred over conservative treatment to restore joint stability and improve functional outcomes. ${ }^{2,5}$ Despite reports of good functional outcomes after PCL reconstruction in the MLIK over the past decades, ${ }^{6}$ this procedure has been associated with some potential problems. These issues are mostly related to the setting of MLIK with the significant surgical morbidity and resultant stiffness, multiple graft usage, and the possibility for tunnel convergence. ${ }^{7,8}$

received

November 3, 2020

accepted

November 29, 2020

published online

January 20, 2021

In recent years, arthroscopic primary PCL repair using modern-day advancements has received renewed attention due to the potential advantages of this procedure over ligament reconstruction. ${ }^{9}$ With arthroscopic primary PCL repair, the ligament can be preserved, thereby avoiding donor-site morbidity, which decreases surgical morbidity, and can allow early mobilization. ${ }^{10,11}$ Additional benefits involve the small diameter of the bone tunnels used for suture passage which result in less problems with tunnel convergence, especially seen in the setting of MLIK. ${ }^{12}$ Given these findings, arthroscopic primary PCL repair may also lead to less complicated revision surgery than PCL reconstruction revision surgery, as is observed during the conversion of failed ACL primary repairs. ${ }^{13}$ Nevertheless, controversies still exist regarding this technique as the current literature is very limited. ${ }^{14}$

(c) 2021. Thieme. All rights reserved. Thieme Medical Publishers, Inc., 333 Seventh Avenue, 18th Floor, New York, NY 10001, USA
DOI https://doi.org/ 10.1055/s-0040-1722695. ISSN 1538-8506. 
The purpose of this review is to discuss the indications and patient selection, different surgical techniques, rehabilitation, and outcomes of modern-day arthroscopic primary PCL repair.

\section{Indications and Patient Selection}

The most important factor for successfully repairing a torn PCL is patient selection, which is mainly based on the tear type and tissue quality of the PCL. In this section, we will therefore discuss the indications, preoperative imaging, and timing of surgery. Since both proximal and distal avulsion tear types may be eligible for surgical repair, both tear types will be discussed in further detail.

\section{Indications}

Learning from both historical and more recent studies focusing on ACL repair, ${ }^{15}$ primary PCL repair should only be performed in patients with proximal or distal tears since the length of tissue remnant must be sufficient to reach back to its anatomical footprint (-Fig. 1).$^{16}$ On the contrary, PCL reconstruction should be performed when patients present with symptomatic midsubstance tears. Besides tear location, it is critical that tissue quality is sufficient to withhold intrasubstance suturing and tensioning toward its femoral or tibial anatomical insertion site. ${ }^{16}$ When taking both tear type and tissue quality into

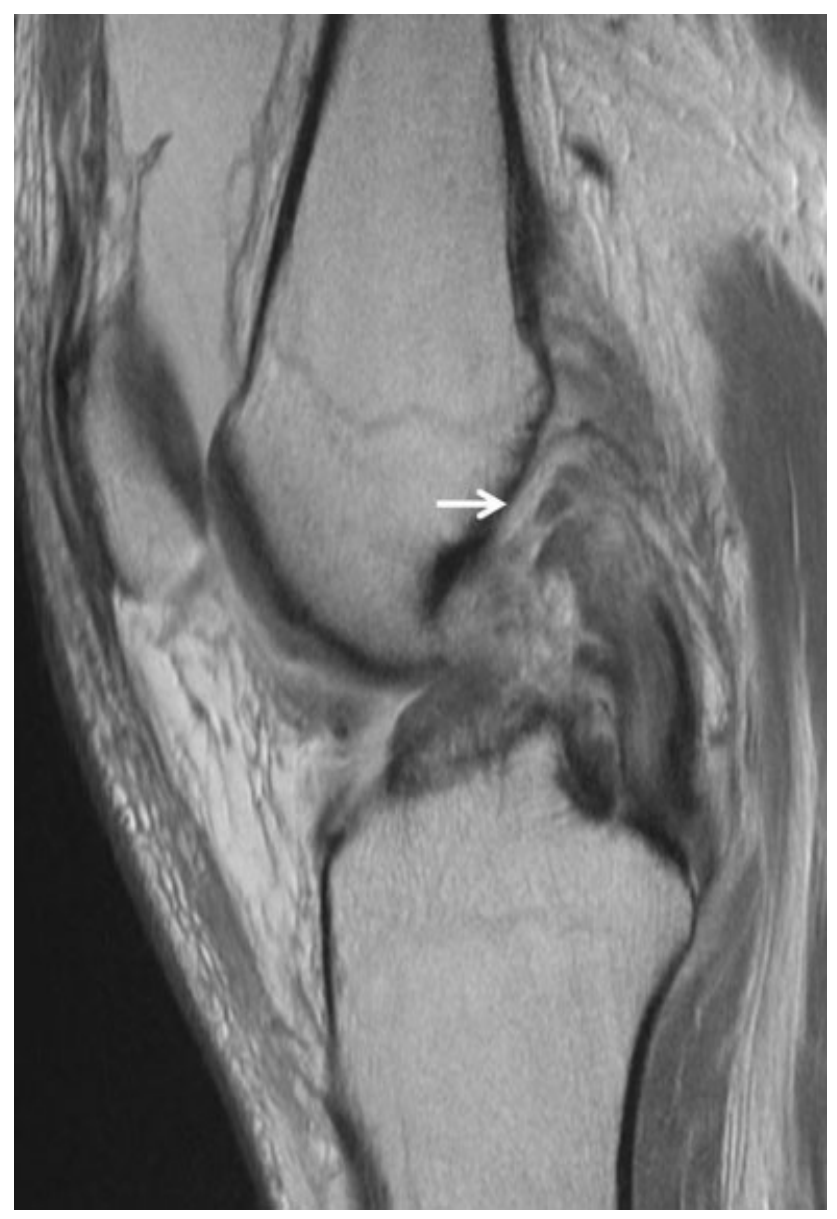

Fig. 1 A sagittal T1-weighted magnetic resonance image shows a proximal (type I) posterior cruciate ligament tear with excellent tissue quality (arrow). account, some studies have suggested that the eligibility for primary PCL repair in the MLIK setting is quite high, with a range from 51 to $73 \%{ }^{17,18}$

\section{Preoperative Imaging}

Although standard radiographs have limited additional value in the diagnoses of acute PCL injuries, radiographs may reveal a displaced distal avulsion fracture or show signs of posterior tibial displacement, ${ }^{19}$ which can be seen in chronic tears. Nevertheless, magnetic resonance imaging (MRI) is the most important study for objectively diagnosing and analyzing PCL tears. ${ }^{20,21}$ Furthermore, advanced imaging can also clearly differentiate between tear types, and therefore, it enables orthopedic surgeons to make a preoperative assessment to predict eligibility for primary PCL repair. ${ }^{22}$ A recent MRI study in MLIK patients attempted to understand this association between tear location and the possibility of repairing proximal PCL tears. ${ }^{21}$ They found that patients were more likely to undergo primary repair if a distal remnant length of more than $41 \mathrm{~mm}$ was present on MRI. It should be noted, however, that the final decision for primary repair is always based upon arthroscopic findings regarding tissue quality.

\section{Timing of Surgery}

Although the optimal time-frame to repair a torn PCL is currently unknown, knee ligament repair surgery is generally preferred within the first weeks postinjury because early surgery is crucial to optimize the likelihood of a successful repair. ${ }^{22}$ After the first several weeks, the ligament can potentially scar and retract, leading to suboptimal tissue quality and tissue length for adequate repair and ligament healing. ${ }^{23}$ Before proceeding with surgery, however, the acutely injured knee should show signs of resolving irritation and improvements in range of motion (ROM). ${ }^{24}$ With this in mind, primary repair-either for isolated tears or in the MLIK setting-is preferably performed between 1 and 4 weeks after injury in the senior author's practice. Future studies, however, are needed to determine the optimal time-frame to perform primary PCL repair.

\section{Surgical Technique}

In this section, the author's surgical preferred technique for primary repair of proximal PCL tears will be discussed first, including two different femoral fixation techniques. Then, we will describe our preferred surgical procedure for distal PCL tears. Finally, other repair techniques that have been reported on in the literature will be briefly reviewed.

\section{Surgical Technique for Proximal Tears}

The patient is placed in the supine position, prepped, and draped in the normal sterile fashion, similar to a PCL reconstruction procedure. Anterolateral and anteromedial portals are created, and a general inspection of the knee joint is performed. Next, the ligamentum mucosum and some of the fat pad is resected to improve visualization. The PCL tear can now be identified and evaluated, and any scar tissue can be 
debrided as necessary by using an arthroscopic shaver. The distal remnant is then reapproximated to its anatomical femoral insertion site with a grasper to assess if sufficient distal remnant length is present. It is important to reduce posterior tibial luxation in the sagittal plane with an anterior drawer force; otherwise, the ligament remnant could appear too short for surgical repair. When tissue length and tissue quality are sufficient to withhold intrasubstance suturing and tensioning to the anatomical wall, the ligament is deemed repairable.

Hence, a small notchplasty can be first performed to both improve the view of the femoral footprint and to induce healing. Suturing of the anterolateral bundle (ALB) starts as distally as possible using the Scorpion Suture Passer (Arthrex, Naples, FL) with a No. 2 FiberWire suture (Arthrex, Naples, FL), and is advanced proximally in an alternating Bunnell-type pattern (-Fig. 2). This procedure is then repeated for the posteromedial bundle (PMB) by using different nonabsorbable sutures. Fixation of the ligament to the femoral wall can now either be performed by using knotless suture anchor technique or using transosseous tunnels in which the sutures are tied over a bone bridge with a button.

When utilizing the suture anchor technique, the anterolateral portal is used for drilling and anchor placement, whereas the anteromedial portal is used as the viewing portal. Two $4.5 \times 20 \mathrm{~mm}$ holes are then drilled, punched, and/or tapped (depending on bone quality) at the native ALB and PMB insertion sides ( - Fig. 3). The PMB is then reattached first with the knee positioned at 90 degrees of flexion by using a $4.75 \mathrm{~mm}$ vented BioComposite SwiveLock (Arthrex, Naples, $\mathrm{FL}$ ), while an anterior drawer force is being applied. The same procedure is then repeated for the ALB although this anchor is preloaded with a high-strain suture FiberTape (InternalBrace, Arthrex, Naples, FL), which acts as the Internal Brace that is felt to be a secondary stabilizer and is thought to allow safer early mobilization in the early phases of rehabili-

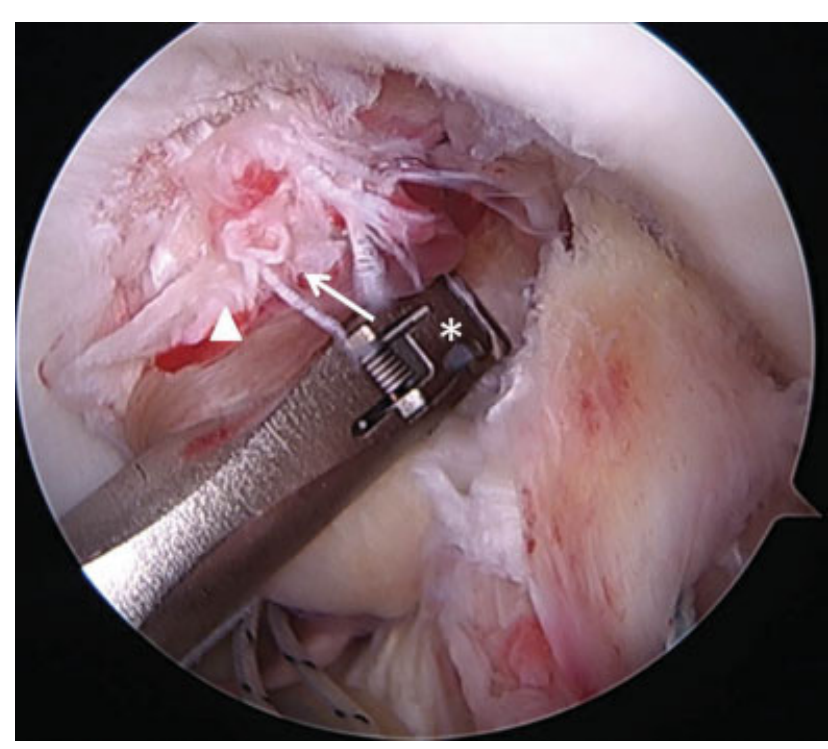

Fig. 2 The distal posterior cruciate ligament remnant (arrowhead) is sutured from distal to proximal (arrow) in a Bunnell-type fashion by using a self-retriever suture-passing device (asterisk).

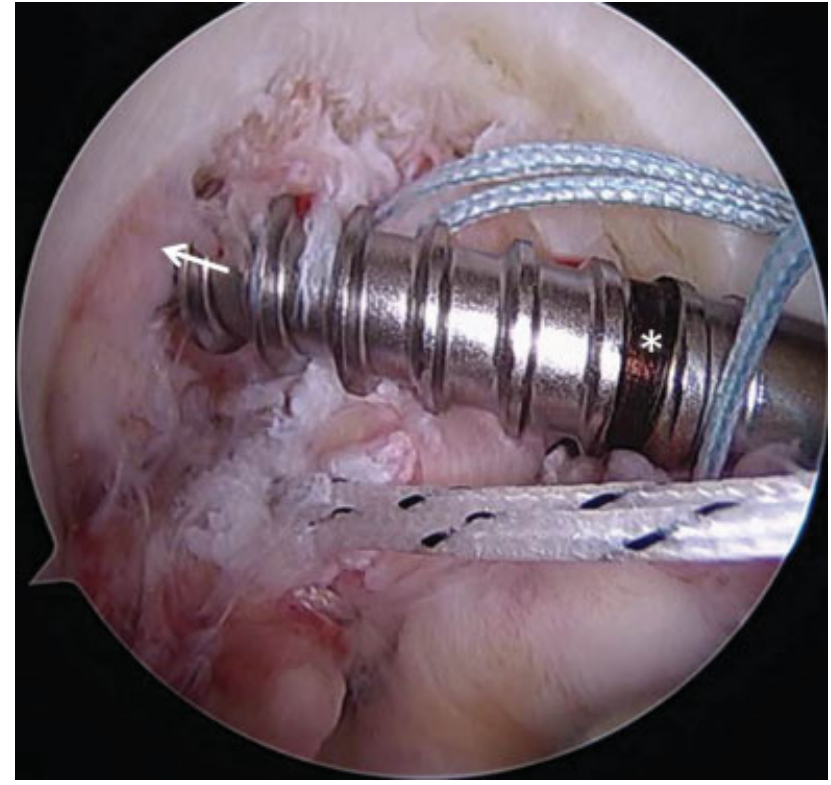

Fig. 3 A bone socket for the first suture anchor is drilled (asterisk) at the footprint of the anterolateral bundle (arrow).

tation. A $2.4 \mathrm{~mm}$ drill pin is then drilled by using a PCL drill guide through the anterolateral tibial cortex up through the tibia and exiting into the center of the tibial PCL insertion. The FiberTape is now retrieved by using a Nitinol passing wire and channeled along the PCL, and passes through the tibial drill hole where it is tensioned and fixed on the anteromedial cortex of the tibia at 90 degrees of flexion by using single suture anchor fixation, thus completing the repair (-Fig. 4).

When utilizing the transosseous tunnel technique, individual drill holes are made through the medial femoral condyle for both the ALB and PMB. Using the PCL femoral guide centered on the anterolateral footprint, a cannulated drill (RetroDrill; Arthrex, Naples, FL) is used to drill from the

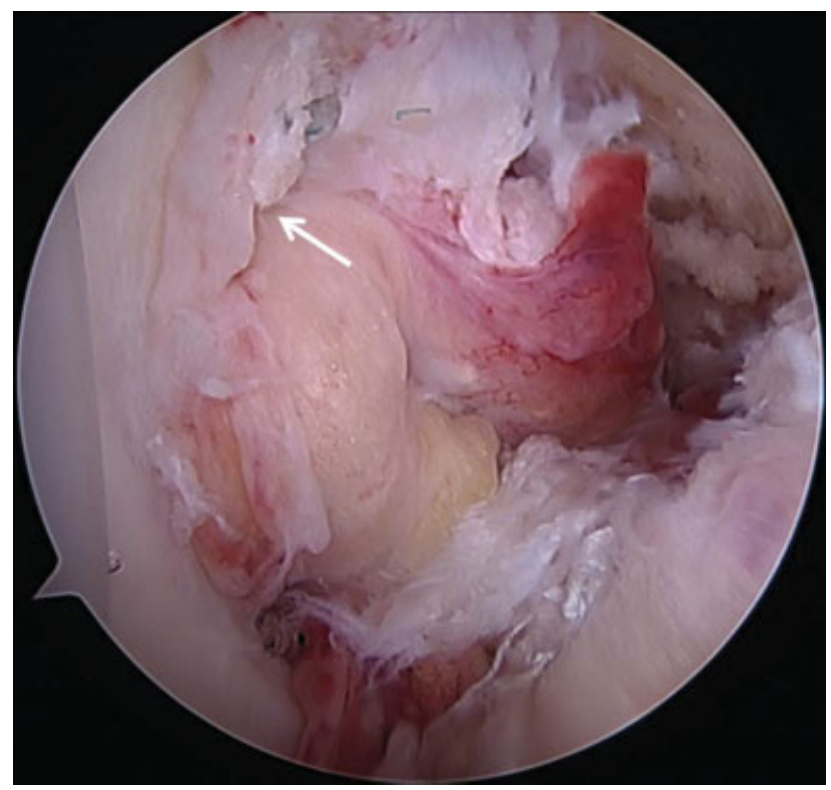

Fig. 4 Primary repair for a proximal posterior cruciate ligament tear is now completed (arrow). 


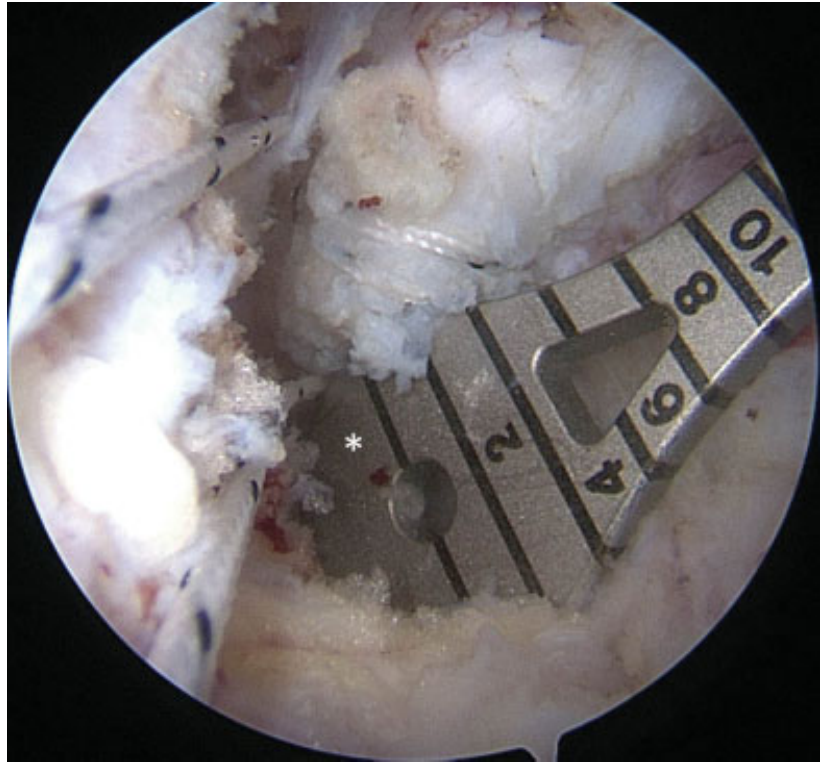

Fig. 5 A posterior cruciate ligament femoral guide (asterisk) is used to drill from the medial side of the femur into the anterolateral footprint to create two separate tunnels.

medial side of the femur into the anterolateral footprint (-Fig. 5). Both sutures are then individually retrieved through each femoral bone tunnel by using a Nitinol passing wire. The ligament remnant is then tensioned back to the femoral wall by tensioning both sutures and applying an anterior drawer force with the knee in 90 degrees flexion. The repair is completed after tying the sutures over the bony bridge by using a ligament button (RetroButton; Arthrex, Naples, FL). Additional suture augmentation can also be applied with this technique by first passing a FiberTape through a Tightrope RT button (Arthrex, Naples, FL). This construct is then passed and tensioned separately through the anterolateral hole where the button is engaged and channeled along with the PCL and through the tibia as discussed above. Fixation and tension of the suture augmentation to the tibia are performed as previously described.

Over the last two decades, multiple arthroscopic primary repair techniques for proximal PCL tears have been proposed. ${ }^{14}$ Nevertheless, most authors have described similar approaches to the transosseous tunnel technique used in the senior author's practice. However, these repair techniques all slightly differ from our surgical procedure as there is variety in the (1) number of independent femoral tunnels drilled, (2) number of individual sutures used, and (3) ligament sutures techniques, such as a locking stitch or Cushing-type stitch. ${ }^{25-29}$ On the contrary, only one additional augmented PCL repair was identified when reviewing the literature. ${ }^{30}$ Hopper et al described a modification of our transosseous tunnel technique using FiberTape augmentation but retrieved the repair stitches proximally through one femoral drill hole.

\section{Surgical Technique for Distal Tears}

In addition to standard anteromedial and anterolateral portal, posteromedial and posterolateral portals are made first in standard fashion to enhance view of the remnant with the

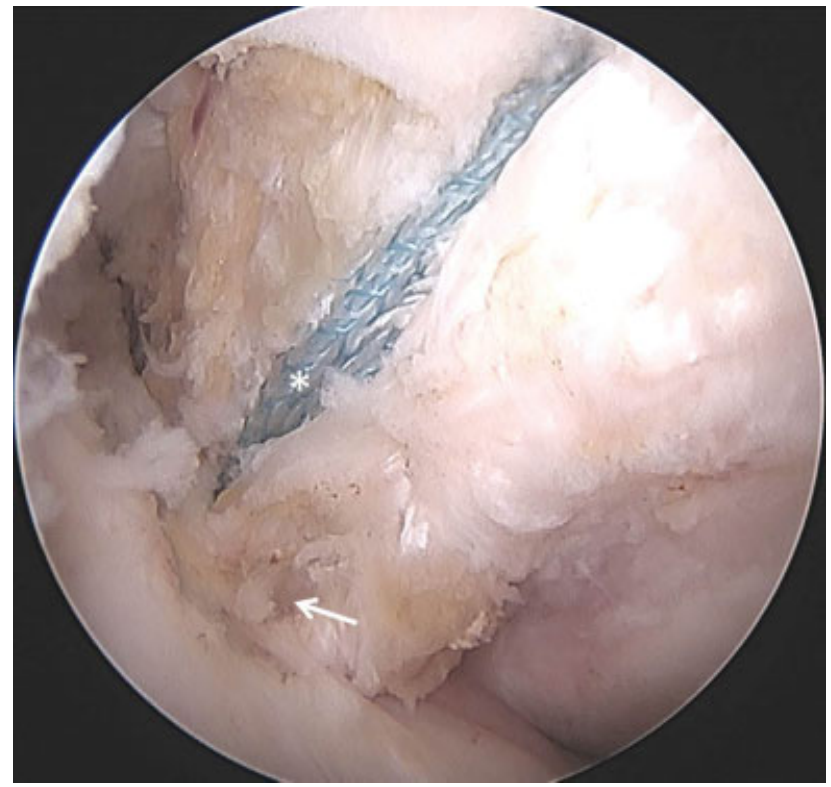

Fig. 6 Primary repair for a distal posterior cruciate ligament tear with suture augmentation (asterisk) is now completed (arrow) in a different patient with a different knee.

scope in the posterolateral portal. The ALB is then sutured by using a Scorpion Suture Passer to pass a locking stitch of FiberWire through the distal remnant. The PLB is sutured in the same manner with the second set of sutures. A cannulated drill is then used to drill two tunnels from the anteromedial side of the tibia into the distal remnant's anatomical insertion site. The sutures of both bundles are now retrieved with a nitinol passer. After applying an anterior drawer force with the knee hold in 90 degrees of flexion, the sutures are tied down over a ligament button and the distal repair is completed (-Fig. 6). An Internal Brace can be added to the distal repair by adding a suture anchor or drill hole at the ALB insertion and modifying the technique described above.

Various arthroscopic and open techniques have been described in the literature for distal avulsion tears. ${ }^{31}$ For bony avulsion tears specifically, open reduction and internal fixation (ORIF) remains the preferred treatment if the bony avulsion is of sufficient size, although it should be noted that there is no consensus regarding the optimal surgical procedure. $^{32}$ These procedures include various techniques, including screws, sutures, K-wires, staples, and toothed plates for bony fixation. ${ }^{32}$

\section{Postoperative Management}

Postoperative rehabilitation protocols are of critical importance for the restoration of full ROM and achieving long-term knee stability. Optimal rehabilitation protocols are injury dependent and based upon the specific injury pattern as most of these injuries are MLIKs.

In general, however, all patients wear a hinged brace after surgery, which is locked in extension during ambulation. It is important to use a brace that places an anterior drawer force onto the proximal tibia to control any posterior tibial translation. Immediate weight bearing and mobilization are allowed 
unless a meniscal repair has been performed, in which partial weight bearing is allowed. ROM exercises are initiated within the first postoperative days but are first limited up to 90 degrees of flexion. Over the course of 6 weeks, this is advanced to full ROM as tolerated by the patient. Closed-chain exercises can be initiated at 6 to 8 weeks postoperatively. Patients can return to perform pivoting sports after 9 to 12 months when muscle strength (comparable to the contralateral leg), ROM, patients' confidence, and sport-specific requirements are sufficient.

\section{Outcomes}

In this section, the outcomes of arthroscopic primary repair for both proximal and distal avulsion tears will be discussed. It is important to note; however, as isolated PCL tears are a rare entity, most outcomes of primary PCL repair have been reported in heterogeneous cohorts in the MLIK setting. Furthermore, several studies have reported on minimally invasive or open PCL repair outcomes, ${ }^{33,34}$ but this will remain out of scope in this review.

\section{Proximal Tears}

Wheatly et al were the first to report on a series of 13 MLIK patients treated with arthroscopic primary repair for femoral avulsed PCL tears. ${ }^{35}$ At a mean follow-up of 4.3 years, excellent results were noted in 11 patients (85\%) that returned for follow-up. International Knee Documentation Committee (IKDC) scores showed normal knee function (grade A) in four patients $(36.4 \%)$, and $7(63.6 \%)$ with nearly normal function (grade B). Furthermore, mean Lysholm and Gillquist scores were 95.4. Finally, all athletes returned to their preinjury or higher level of sports competition.

In another small case series, Ross et al reported the outcomes of five selected patients who were treated with arthroscopic primary PCL repair in the MLIK setting. ${ }^{25}$ At latest follow-up, the authors described successful healing of the PCL with a grade 1 or less posterior drawer in four patients (80\%), while there was one clinical failure.

Similar outcomes were reported by Giordano et al, as they reported on three patients who underwent arthroscopic primary PCL repair. ${ }^{26}$ In their study, all patients achieved clinical stability, full ROM and returned to their preinjury competitive level at a mean follow-up of 2 years. Furthermore, the IKDC score was normal in two of the patients and nearly normal in the third.

Heusdens et al presented in 2019 the 2-year outcomes of two patients who underwent arthroscopic PCL repair with suture augmentation. ${ }^{36}$ At final follow-up, both patients had excellent clinical results with full ROM, IKDC scores of 83 and 100 , Lysholm's scores of 99 and 100 , and had returned to their preinjury sports level. Additionally, MRI showed a healed and tensioned PCL in both cases.

Vermeijden et al reported the short-term outcomes of a heterogeneous cohort of 48 patients with MLIK. ${ }^{18}$ of all patients, 27 (56\%) presented with a PCL tear, of which 19 (73\%) were repaired. At a mean follow-up of 2.5 years, the PCL repair failed in three patients (17\%), of which two underwent ligament reconstruction and one was treated conservatively. However, no other clinical or functional outcomes were described in this study.

Lastly, two recent case reports reported good outcomes following arthroscopic PCL repair in the MLIK setting with concomitant repairs of the ACL and MCL. ${ }^{37}$ Murakimi et al reported that at 2 years after surgery, their patient had a clinically stable knee, and the Lysholm, Knee Injury and Osteoarthritis Outcome Score (KOOS) symptoms, KOOS pain, KOOS activities of daily living, KOOS sport, and KOOS quality of life scores were good, respectively. KT-2000 revealed a mean side-to-side difference of $1.2 \mathrm{~mm}$. Moreover, MRI showed that the PCL fibers were in continuity and under tension.

Zhao et al recently presented the case of 9-year-old patient treated with primary PCL repair. ${ }^{38}$ At 2-year follow-up, the patient was satisfied with his operated knee, and physical examination showed a negative posterior drawer test and full ROM. The postoperative Lysholm's score was 85 . Finally, MRI showed consolidation of the bony fragment.

\section{Distal Tears}

In 2010, Kim et al described the outcomes of six pediatric or adolescent patients (mean age $=12.3$ years) treated with arthroscopic primary repair for distal PCL soft-tissue avulsion tears. ${ }^{39}$ At a mean follow-up of 3.1 years, mean side-to-side difference measured with KT-2000 arthrometer was $2.3 \mathrm{~mm}$. In addition, the mean Lysholm's score was 95, and two patients had a normal IKDC, three had a nearly normal score, while one had an abnormal score. Finally, five of the six patients returned to their preinjury level of sports activities.

When specifically only looking at studies focusing on tibialsided bony avulsion tears, a recent systematic review identified 10 studies reporting on 215 patients treated with various fixation techniques but all via an arthroscopic approach. ${ }^{32}$ In this study, $90 \%$ had a negative or grade 1 posterior drawer test. Additionally, the mean Lysholm's score was 95, while 78.9\% reported a normal knee, $17.6 \%$ a nearly normal knee, and 3.5\% an abnormal knee according to their IKDC score. Furthermore, mean Tegner's activity score decreased from 7.1 preinjury to 6.8 postinjury.

More recently, Yoon et al reviewed the clinical outcomes of 18 patients treated for tibial avulsion fractures using a transosseous arthroscopic suture bridge technique at a mean follow-up of 2.2 years. ${ }^{40}$ No failures were reported. In addition, mean Lysholm's score was 90 and the IKDC score was normal in 10 patients (56\%), nearly normal in seven (39\%) and abnormal in one (5\%). All patients achieved full ROM, and radiographic evaluation showed solid union at the fracture site in all 18 cases.

\section{Pooled Outcomes}

When pooling all 269 patients using standardized methods, ${ }^{41}$ the mean failure rate was $1 \%$, reoperation rate was $6 \%$, and complication rate was $1 \%$. In addition, the mean Lysholm's score was $94 \pm 4$, IKDC subjective was $96 \pm 3$, and $96 \%$ of patients had a normal or near-normal knee function according to the IKDC objective score. Furthermore, 97\% of patients reached their preinjury level of activity level, while the 
言咅要

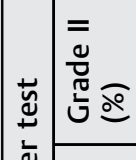

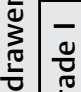

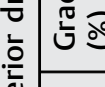

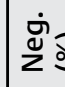

\begin{tabular}{|c|c|c|c|c|c|c|c|c|c|c|c|c|c|}
\hline 0 & 0 & શ & 0 & 0 & 0 & 0 & 0 & 0 & & 0 & 0 & 6 & \\
\hline 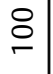 & 0 & & เ & 0 & 0 & $\theta$ & 0 & $\stackrel{ \pm}{\square}$ & & $\stackrel{n}{\leftarrow}$ & $m$ & 요 & Ln \\
\hline 0 & 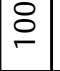 & & ๖े & ○ & ○ & ஜ & ○ & $\infty$ & $\mathscr{\bullet}$ & $\infty$ & á & F & นั \\
\hline
\end{tabular}

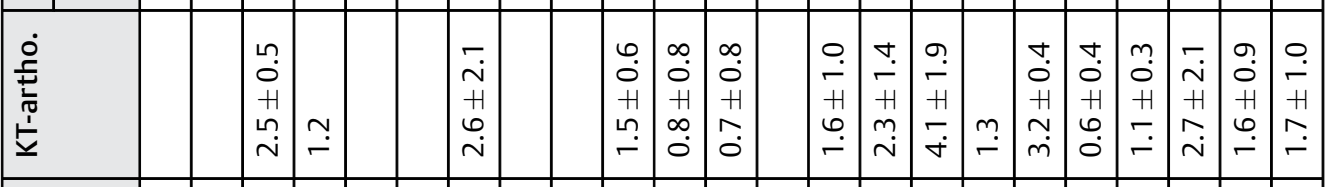

产豈 $\quad$ वे

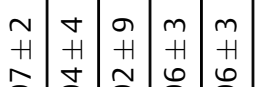

든

䓂㝘导

$\therefore \quad \div \div \div \% \cong$

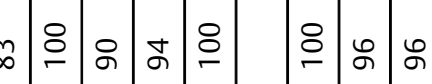

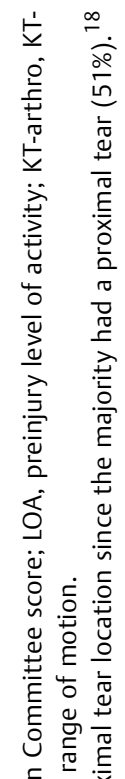

는

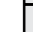

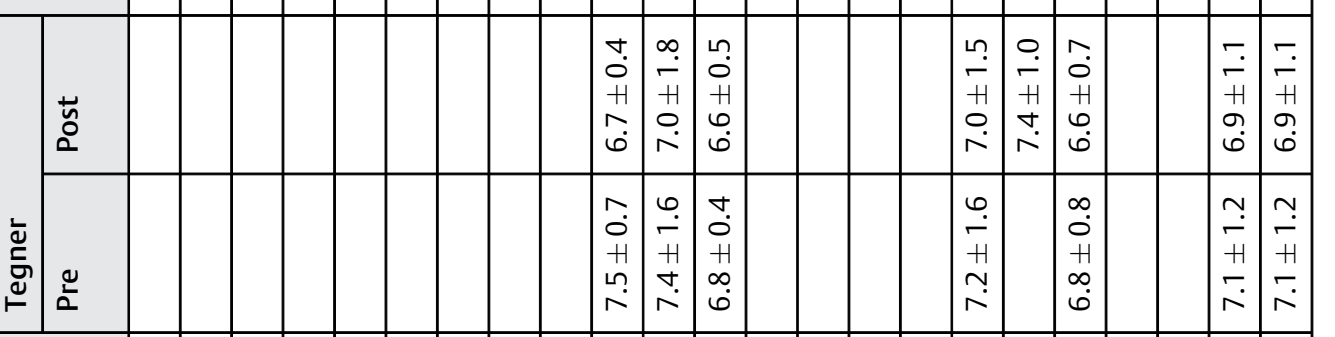

$\frac{E}{\frac{E}{0}}$

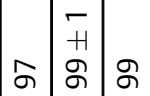

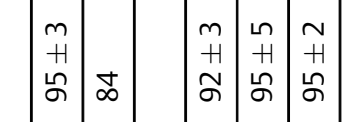

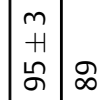

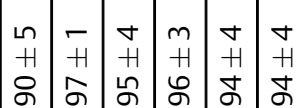

可

-

음

ஃ

$\therefore$ ○

음 ลे

हे

कृष

000

$\circ 0$

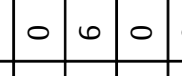

$\pm$

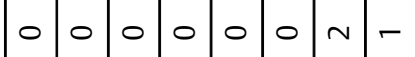

产

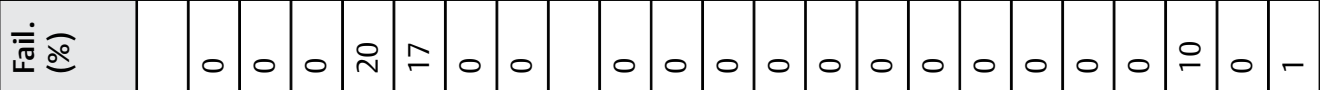

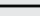

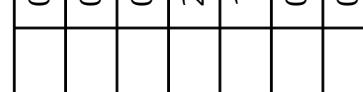

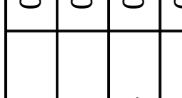

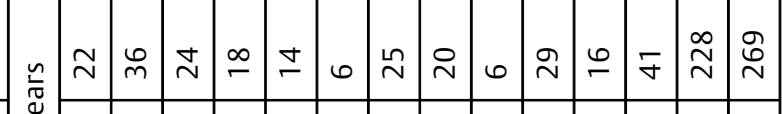

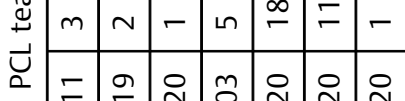

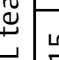

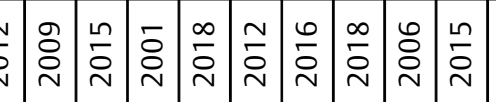

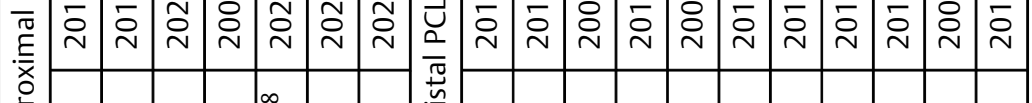

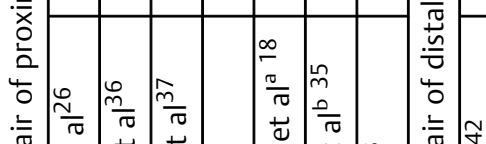

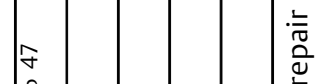

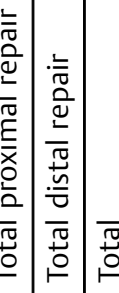


Tegner's score changed from $7.1 \pm 1.2$ to $6.9 \pm 1.1$. Finally, the mean posterior tibial translation was $1.7 \pm 1.0$, with $90 \%$ of patients having a negative posterior drawer test and $93 \%$ achieving full ROM at latest follow-up. When specifically looking at proximal and distal tears only, it was noted that considerably more data currently is available for primary repair of distal tears ( 41 vs. 228 patients, respectively). Nevertheless, failure rates in both groups were low (between and 0 and $10 \%$, while functional outcomes were noted to be good with mean Lysholm's and IKDC subjective scores around 90 (-Table 1).

\section{Conclusion}

Over the last decade, primary PCL repair using arthroscopy has seen a resurgence of interest. When only performing this surgery in patients with proximal or distal avulsion type tears, good outcomes can be expected by following arthroscopic primary PCL repair. Although the current literature regarding primary PCL repair is not extensive and most studies have focused on heterogeneous cohorts of MLIKs, the pooled outcomes of arthroscopic repair are excellent in patients with proximal and distal tears with low failure rates and good subjective outcomes and objective stability. We recommend that arthroscopic primary PCL repair should be a tool in the armamentarium of orthopedic surgeons treating patients with isolated PCL tears or PCL tears in the MLIK setting.

\section{Funding}

G.S.D. declares that he is a paid consultant and receives research grants from Arthrex (Naples, FL).

\section{Conflict of Interest}

None declared.

\section{References}

1 Satku K, Chew CN, Seow H. Posterior cruciate ligament injuries. Acta Orthop Scand 1984;55(01):26-29

2 LaPrade CM, Civitarese DM, Rasmussen MT, LaPrade RF. Emerging updates on the posterior cruciate ligament: a review of the current literature. Am J Sports Med 2015;43(12):3077-3092

3 Vaquero-Picado A, Rodríguez-Merchán EC. Isolated posterior cruciate ligament tears: an update of management. EFORT Open Rev 2017;2(04):89-96

4 Howells NR, Brunton LR, Robinson J, Porteus AJ, Eldridge JD, Murray JR. Acute knee dislocation: an evidence based approach to the management of the multiligament injured knee. Injury 2011;42(11):1198-1204

5 Noyes FR, Barber-Westin SD. Posterior cruciate ligament injuries. In: Noyes FR, Barber-Westin SD, eds. Noyes' Knee Disorders: Surgery, Rehabilitation, Clinical Outcomes. Philidelphia: Elsevier; 2017:447-526

6 Pache S, Aman ZS, Kennedy M, et al. Posterior cruciate ligament: current concepts review. Arch Bone Jt Surg 2018;6(01):8-18

7 Poehling-Monaghan KL, Salem H, Ross KE, et al. Long-term outcomes in anterior cruciate ligament reconstruction: a systematic review of patellar tendon versus hamstring autografts. Orthop J Sports Med 2017;5(06):2325967117709735

8 Paschos NK, Howell SM. Anterior cruciate ligament reconstruction: principles of treatment. EFORT Open Rev 2017;1(11):398-408
9 Lissy M, Dy CJ, Ranawat AS, DiFelice GS. Arthroscopic Primary cruciate repair in the multiligament injured knee. 2013:193-210

10 van der List JP, DiFelice GS. Range of motion and complications following primary repair versus reconstruction of the anterior cruciate ligament. Knee 2017;24(04):798-807

11 van der List JP, DiFelice GS. Primary repair of the anterior cruciate ligament: a paradigm shift. Surgeon 2017;15(03):161-168

12 Moatshe G, Chahla J, LaPrade R, Engebretsen L. Diagnosis and treatment of multiligament knee injury: state of the art. J ISAKOS Jt Disord Orthop Sport Med 2017;2(03):152-161

13 van der List JP, Vermeijden HD, O'Brien R, DiFelice GS. Anterior cruciate ligament reconstruction following failed primary repair: surgical technique and a report of three cases. Minerva Ortop Traumatol 2019;70(02):70-77

14 Vermeijden HD, van der List JP, DiFelice GS. Arthroscopic posterior cruciate ligament primary repair. Sports Med Arthrosc Rev 2020; 28(01):23-29

15 van der List JP, DiFelice GS. Preservation of the anterior cruciate ligament: a treatment algorithm based on tear location and tissue quality. Am J Orthop 2016;45(07):E393-E405

16 van der List JP, DiFelice GS. Preoperative magnetic resonance imaging predicts eligibility for arthroscopic primary anterior cruciate ligament repair. Knee Surg Sports Traumatol Arthrosc 2018;26(02):660-671

17 Twaddle BC, Bidwell TA, Chapman JR. Knee dislocations: where are the lesions? A prospective evaluation of surgical findings in 63 cases. J Orthop Trauma 2003;17(03):198-202

18 Vermeijden HD, Jonkergouw A, van der List JP, DiFelice GS. The multiple ligament-injured knee: when is primary repair an option? Knee 2020;27(01):173-182

19 Shino K, Mitsuoka T, Horibe S, Hamada M, Nakata K, Nakamura N. The gravity sag view: a simple radiographic technique to show posterior laxity of the knee. Arthroscopy 2000;16(06):670-672

20 Laoruengthana A, Jarusriwanna A. Sensitivity and specificity of magnetic resonance imaging for knee injury and clinical application for the Naresuan University Hospital. J Med Assoc Thai 2012; 95(Suppl 10):S151-S157

21 Goiney CC, Porrino J, Twaddle B, Richardson ML, Mulcahy H, Chew FS. The value of accurate magnetic resonance characterization of posterior cruciate ligament tears in the setting of multiligament knee injury: imaging features predictive of early repair vs reconstruction. Curr Probl Diagn Radiol 2016;45(01):10-16

22 DiFelice GS, van der List JP. Arthroscopic primary repair of posterior cruciate ligament injuries. Oper Tech Sports Med 2015;23(04):307-314

23 Murray MM, Martin SD, Martin TL, Spector M. Histological changes in the human anterior cruciate ligament after rupture. J Bone Joint Surg Am 2000;82(10):1387-1397

24 Balcarek P, Sawallich T, Walde TA, et al. [Influence of cyclops syndrome after anterior cruciate ligament reconstruction on the functional outcome]. Sportverletz Sportschaden 2008;22(04): 220-224

25 Ross G, Driscoll J, McDevitt E, Scheller A Jr. Arthroscopic posterior cruciate ligament repair for acute femoral "peel off" tears. Arthroscopy 2003;19(04):431-435

26 Giordano BD, Dehaven KE, Maloney MD. Acute femoral "peel-off" tears of the posterior cruciate ligament: technique for arthroscopic anatomical repair. Am J Orthop 2011;40(05):226-232

27 Rosso F, Bisicchia S, Amendola A. Arthroscopic repair of "peel-off" lesion of the posterior cruciate ligament at the femoral condyle. Arthrosc Tech 2014;3(01):e149-e154

28 Rugg CM, Liu T, Zhang AL. Arthroscopic primary bundle-specific posterior cruciate ligament repair with transosseous fixation. Arthrosc Tech 2019;8(08):e911-e916

29 Pisanu G, Moura JL, Saithna A, Sonnery-Cottet B. Arthroscopic repair of proximal posterior cruciate ligament injuries in pediatric patients. Arthrosc Tech 2019;8(07):e691-e695 
30 Hopper GP, Heusdens CHW, Dossche L, Mackay GM. Posterior cruciate ligament repair with suture tape augmentation. Arthrosc Tech 2018;8(01):e7-e10

31 Katsman A, Strauss EJ, Campbell KA, Alaia MJ. Posterior cruciate ligament avulsion fractures. Curr Rev Musculoskelet Med 2018;11 (03):503-509

32 Hooper PO III, Silko C, Malcolm TL, Farrow LD. Management of posterior cruciate ligament tibial avulsion injuries: a systematic review. Am J Sports Med 2018;46(03):734-742

33 Owens BD, Neault M, Benson E, Busconi BD. Primary repair of knee dislocations: results in 25 patients ( 28 knees) at a mean follow-up of four years. J Orthop Trauma 2007;21(02):92-96

34 Heitmann M, Akoto R, Krause M, et al. Management of acute knee dislocations: anatomic repair and ligament bracing as a new treatment option-results of a multicentre study. Knee Surg Sports Traumatol Arthrosc 2019;27(08):2710-2718

35 Wheatley WB, Martinez AE, Sacks T, et al. Arthroscopic posterior cruciate ligament repair. Arthroscopy 2002;18(07):695-702

36 Heusdens CHW, Tilborghs S, Dossche L, Van Dyck P. Primary posterior cruciate ligament repair with the novel suture tape augmentation technique. Surg Technol Int 2019;34(34):469-475

37 Murakami R, Honda E, Fukai A, Yoshitomi H, Sanada T, Iwaso H. Single-stage arthroscopic anterior and posterior cruciate ligament repairs and open medial collateral ligament repair for acute knee dislocation. Case Rep Orthop 2020;2020:7348201

38 Zhao X, da Kuang S, Su C, Feng XW, Hua LG, Guang GS. Arthroscopic treatment of femoral avulsion fracture of the posterior cruciate ligament in association with meniscus tear. Orthop Surg 2020;12(02):

39 Kim SJ, Jo SB, Kim SG, Park IS, Kim HP, Kim SH. Peel-off injury at the tibial attachment of the posterior cruciate ligament in children. Am J Sports Med 2010;38(09):1900-1906

40 Yoon JR, Park CD, Lee DH. Arthroscopic suture bridge fixation technique with multiple crossover ties for posterior cruciate ligament tibial avulsion fracture. Knee Surg Sports Traumatol Arthrosc 2018;26(03):912-918
41 Higgins J, Green S. Cochrane Handbook for Systematic Reviews of Interventions. Accessed 2020 at: https://training.cochrane.org/cochrane-handbook-systematic-reviews-interventions

42 Chen LB, Wang H, Tie K, Mohammed A, Qi YJ. Arthroscopic fixation of an avulsion fracture of the tibia involving the posterior cruciate ligament: a modified technique in a series of 22 cases. Bone Joint J 2015;97-B(09):1220-1225

43 Chen SY, Cheng CY, Chang SS, et al. Arthroscopic suture fixation for avulsion fractures in the tibial attachment of the posterior cruciate ligament. Arthroscopy 2012;28(10):1454-1463

44 Gui J, Wang L, Jiang Y, Wang Q, Yu Z, Gu Q. Single-tunnel suture fixation of posterior cruciate ligament avulsion fracture. Arthroscopy 2009;25(01):78-85

45 Huang W, Gong X, Rahul M, et al. Anterior arthroscopic-assisted fixation of posterior cruciate ligament avulsion fractures. Eur J Med Res 2015;20(01):88

46 Kim SJ, Shin SJ, Choi NH, Cho SK. Arthroscopically assisted treatment of avulsion fractures of the posterior cruciate ligament from the tibia. J Bone Joint Surg Am 2001;83(05):698-708

47 Pardiwala DN, Agrawal D, Patil V, Saini U, Dhawal P. Paper 133: comparison of open versus arthroscopic fixation for isolated PCL tibial bony avulsions: a prospective randomized study with minimum 2 year follow-up. Arthrosc J Arthrosc Relat Surg 2012;28(09):e413-e414

48 Sabat D, Jain A, Kumar V. Displaced posterior cruciate ligament avulsion fractures: A retrospective comparative study between open posterior approach and arthroscopic single-tunnel suture fixation. Arthroscopy 2016;32(01):44-53

49 Zhao J, He Y, Wang J. Arthroscopic treatment of acute tibial avulsion fracture of the posterior cruciate ligament with suture fixation technique through Y-shaped bone tunnels. Arthroscopy 2006;22(02):172-181

50 Zhu W, Lu W, Cui J, et al. Treatment of tibia avulsion fracture of posterior cruciate ligament with high-strength suture fixation under arthroscopy. Eur J Trauma Emerg Surg 2017;43(01): 137-143 